\title{
Adherencia al tratamiento de pacientes con artritis reumatoidea que reciben medicamentos biológicos
}

\author{
Rafael Chaparro del Moral', Oscar Luis Rillo, Mariana Benegas ${ }^{1}$, María de los Angeles Correa², Gustavo Citera ${ }^{2}$, \\ José A Maldonado Cocco $^{2}$, Gustavo Casado ${ }^{3}$, Victor Caputo ${ }^{3}$, Claudia Helling ${ }^{4}$, M Natalia Tamborenea ${ }^{4}$, Eduardo \\ Mysler ${ }^{4}$, Guillermo Tate ${ }^{4}$, Lorena Takashima ${ }^{5}$, Anastasia Secco ${ }^{5}$, Mariana Salcedo ${ }^{6}$, Andrea Baños ${ }^{7}$, Patricia \\ Cowan ${ }^{7}$, Josefina Molina ${ }^{8}$, Emilia Cavillon ${ }^{9}$, Ida Exeni ${ }^{10}$, Carla Gobbi1 ${ }^{11}$, Darío Scublinsky ${ }^{12}$, Mónica Diaz ${ }^{13}$ \\ Grupo de Estudio de Artritis Reumatoidea de la SAR (GESAR-AR) \\ ${ }^{1}$ Hospital General de Agudos Dr. Enrique Tornú, CABA. ${ }^{2}$ Instituto de Rehabilitación Psicofísica, CABA. ${ }^{3}$ Hospital Militar Central, CABA. \\ ${ }^{4}$ Organización Médica de Investigación, CABA. ${ }^{5}$ Hospital Rivadavia, CABA. ${ }^{6}$ Consultorio particular, San Nicolás - Buenos Aires. ${ }^{7}$ Consultorio \\ particular, CABA. ${ }^{8}$ Consultorio particular, Yerba Buena - Tucumán. ${ }^{9}$ Hospital Italiano de Córdoba, Córdoba. ${ }^{10}$ Hospital de Córdoba, Córdoba. \\ ${ }^{11}$ Cátedra de Medicina II, Córdoba. ${ }^{12}$ Primera Cátedra de Farmacología, UBA, CABA. ${ }^{13}$ Hospital Zonal Bariloche, Bariloche.
}

\begin{abstract}
Resumen
Introducción: Al igual que en otras enfermedades crónicas, la adherencia al régimen terapéutico de los pacientes con artritis reumatoidea (AR) es baja (entre 30 y 80\%), dependiendo de la definición de adherencia y de la metodología empleada para medirla. En este estudio se propone determinar el nivel de adherencia al tratamiento en pacientes con AR que reciben DMAR biológicas e identificar factores asociados a la falta de cumplimiento a la terapia.
\end{abstract}

Material y métodos: Se realizó un estudio analítico, observacional de corte transversal en donde se incluyeron pacientes consecutivos con AR según criterios de clasificación (ACR'87) que se encontraban recibiendo fármacos biológicos para el tratamiento de su enfermedad en los últimos seis meses y que asistieron a la consulta ambulatoria. Para la valoración de la adherencia a DMAR se utilizaron los cuestionarios CQR (Compliance Questionnaire on Rheumatology) y el cuestionario SMAQ (Simplified Medication Adherence Questionnaire).

Resultados: Se encuestaron 345 pacientes. Mediante el cuestionario SMAQ se observó una adherencia del 50\% (159 pacientes). El Cuestionario CQR tuvo un puntaje mediano de 78 puntos (RIC 67-86). El $47 \%$ (147 pacientes) fueron adherentes (CQR >80). Sobre los pacientes incluidos, 151 (48\%) refirieron no haber tenido ningún retraso, pérdida 0 adelanto de la dosis del biológico en los últimos 6 meses de tratamiento. El 52\% no adherentes tuvo como causas: 146 (46\%) pérdida de al menos una dosis del biológico con una mediana de dosis perdidas de 2 (RIQ: 1-3); 117 (37\%) tuvo al menos un retraso en las dosis del biológico y 8 (2\%) adelantó la dosis. Los factores asociados al no cumplimiento de la terapia biológica fueron el tipo de cobertura médica, que el paciente no haya notado mejoría y la esperanza de una rápida respuesta al tratamiento, y la falta de adherencia a DMAR.

\section{Abstract}

Introduction: As in other chronic diseases, adherence to the treatment regimen of patients with rheumatoid arthritis (RA) is low (between 30 and $80 \%$ ), depending on the definition of adherence and the methodology used to measure it. This study aims to determine the level of adherence to treatment in patients with RA receiving biological DMARDs and identify factors associated with non-compliance to therapy.

Methods: An analytical cross-sectional study was conducted. RA patients were included sequentially according to ACR' 87 classification criteria. All of them were receiving biologics during the last six months and attended the outpatient's clinics. To assess adherence to DMARDs, CQR (Compliance Questionnaire on Rheumatology) and SMAQ (Simplified Medication Adherence Questionnaire) were used.

Results: Three hundred and forty five patients were surveyed. Through SMAQ it was observed a 50\% adherence (159 patients. CQR had a median score of 78 points (IQR 67-86). Forty seven percent (147 patients) had adherence (CQR $>80)$. Given the included patients, 151 (48\%) reported not having had any delay, loss or advancement of the biological dose in the last 6 months of treatment. Fifty two percent had no adherents as causes: $146(46 \%)$ loss of at least one dose of biological dose with a median loss of 2 (IQR 1-3), $117(37 \%)$ had at least a delay in the dose of biological and $8(2 \%)$ doses forward. Factors associated with noncompliance of biological therapy were the type of medical coverage, the patient has not noticed improvement and hope for a quick response to treatment, and lack of adherence to DMARDs.

Key words: adherence, compliance, DMARDs, biologics.

Palabras clave: adherencia, cumplimiento, DMAR, biológicos.

\section{Correspondencia}

E-mail: drchaparro@hotmail.com 


\section{Introducción}

La adherencia (o el cumplimiento y persistencia) a un régimen de tratamiento farmacológico se refiere a si el paciente recibe el fármaco acorde a las instrucciones prescriptas ${ }^{1}$.

La falta de adherencia se observa en una gran proporción de pacientes con enfermedades crónicas, siendo los estudios más relevantes los que fueron realizados en pacientes con HIV, TBC y hepatitis $\mathrm{C}^{2,3}$. Al menos el 50\% de los adultos a los que se les prescriben fármacos para condiciones crónicas tendrán dificultades para continuar con su régimen terapéutico después de seis meses de tratamiento ${ }^{4}$.

La pobre adherencia contribuye a un resultado terapéutico desfavorable e incrementa la utilización y los costos en servicios de salud ${ }^{5}$. La Organización Mundial de la Salud (OMS) reconoce este problema a nivel global con una mayor preocupación sobre países subdesarrollados ${ }^{6}$.

La falta de adherencia es un fenómeno conocido en todas las áreas terapéuticas, que contribuye a una respuesta inadecuada o al fracaso del tratamiento, al empeoramiento y la progresión de la enfermedad y a las recaídas o reaparición de los síntomas ${ }^{7,8,9}$.

\section{Antecedentes en Artritis Reumatoidea:}

Las drogas modificadoras de la artritis reumatoidea (DMAR) de origen biológico han superado los resultados obtenidos con DMAR convencionales en ensayos clínicos aleatorios $(\mathrm{ECA})^{10,11,12}$. Sin embargo, en la práctica habitual los resultados podrían ser diferentes de aquellos obtenidos en los ECA. Aunque los ECA sean considerados 'el patrón oro' para evaluar intervenciones terapéuticas, los mismos tienen ciertas limitaciones a la hora de generalizar sus resultados a la práctica médica habitual tales como: duración relativamente corta, dificultades en la detección de efectos adversos de baja frecuencia, poblaciones homogéneas, mayor control del cumplimiento del tratamiento, flexibilidad limitada para ajustes de la medicación y restricciones para tratamientos concomitantes o con otras DMAR diferentes a metotrexato ${ }^{13,14,15}$.

Al igual que en otras enfermedades crónicas, la adherencia al régimen terapéutico de los pacientes con Artritis Reumatoidea es baja (entre 30 y $80 \%$ ), dependiendo de la definición de adherencia y de la metodología empleada para medirla ${ }^{16}$. La adherencia autorreferida (evaluada mediante cuestionarios) a las DMAR no biológicas es de un 60-68\% según el instrumento de medición ${ }^{17}$.

Los datos disponibles sobre la adherencia de pacientes a las DMAR tradicionales conllevan la preocupación de que exista una pobre adherencia a DMAR biológicas y que, debido a esto, la eficacia que las DMAR biológicas han demostrado en los ECA sea inferior en la práctica diaria. Esto puede verse incrementado en países como el nuestro, donde la heterogeneidad en la distribución de los recursos podría hacer peligrar la continuidad de las terapias biológicas para cierto grupo de pacientes.

En este estudio se propone determinar el nivel de adherencia al tratamiento en pacientes con AR que reciben DMAR biológicas e identificar factores asociados a la falta de cumplimiento a la terapia.

\section{Objetivos}

Nos propusimos: a) Evaluar el cumplimiento al tratamiento de pacientes con artritis reumatoidea que se encuentran recibiendo terapia biológica, b) Identificar factores asociados a la falta de adherencia.

\section{Materiales y métodos}

Definiciones de adherencia, cumplimiento y persistencia Existe una gran variación en las definiciones de adherencia, cumplimiento y persistencia.

El cumplimiento es "el grado con el cual un paciente actúa conforme al intervalo, dosis, y régimen de medicación prescrito". Típicamente es expresado como porcentaje del número total de dosis tomadas (para estudios prospectivos) o días de terapia disponible (evaluación retrospectiva), en relación con el período de tiempo de observación durante la cual es medido el cumplimiento"18. En estudios de corte transversal realizados mediante cuestionarios que evalúan el cumplimiento autorreferido, se considera como sinónimo de adherencia a un porcentaje de respuestas positivas del $80 \%$ o más.

La persistencia de medicación es "el tiempo desde el inicio a la discontinuación de la terapia y es medida en las unidades de tiempo" ${ }^{19}$. Además, los términos supervivencia de la droga, retención o continuación del tratamiento a menudo son usados para describir persistencia.

La adherencia incluye ambos aspectos de la toma de un medicamento: el cumplimiento (que incluye regularidad y continuidad) y la persistencia ${ }^{19}$.

\section{Métodos para evaluar adherencia}

Existe un solo cuestionario validado en Reumatología, conocido como CQR de las siglas en inglés "Compliance Questionnaire on Rheumatology". El CQR mide adherencia autorreferida a regímenes de tratamiento e 
identifica factores que contribuyen a un cumplimiento subóptimo. Este cuestionario de adherencia CQR tiene una sensibilidad y especificidad del $98 \%$ y $67 \%$ para detectar buena adherencia al tratamiento ${ }^{20}$ y consta de 19 ítems los cuales el paciente responde indicando cuál es su nivel de acuerdo con el enunciado. En Argentina, este cuestionario fue validado a nuestra lengua recientemente por reumatólogos del IREP.

Para la valoración de la adherencia a DMAR además del CQR se emplea el cuestionario SMAQ (Simplified Medication Adherence Questionnaire), que consta de seis preguntas y ha sido validado en España, presentando un $72 \%$ de sensibilidad y $91 \%$ de especificidad. El mismo clasifica como no adherente ${ }^{2}$.

Se consideró falta de adherencia a DMAR biológica a cualquier alteración de la prescripción o dosificación médica: ya sea retraso, pérdida o adelanto de la dosis del medicamento biológico.

\section{Diseño metodológico}

Se realizó un estudio analítico, observacional de corte transversal en donde se incluyeron pacientes consecutivos con AR según criterios de clasificación (ACR'87) ${ }^{21}$ que se encontraban recibiendo fármacos biológicos para el tratamiento de su enfermedad en los últimos seis meses y que asistieron a la consulta ambulatoria en los diferentes centros participantes, durante el período comprendido entre noviembre de 2010 y abril de 2011. La recolección de los datos se realizó mediante encuesta autorrespondida.

Se recolectaron datos sociodemográficos (edad, sexo, nivel de educación, cobertura social), tiempo de evolución de la $\mathrm{AR}$, número de medicamentos que toma para $\mathrm{AR}$, número de medicamentos que toma por cualquier otra causa, medicamento biológico indicado, frecuencia de administración, fecha de inicio del tratamiento biológico, forma de administración, retrasos en la administración, pérdida de dosis del biológico, posibles preocupaciones o limitaciones en relación a la terapia biológica (vía y frecuencia de administración, rapidez del efecto, efectos adversos, percepción de riesgo-beneficio, percepción de eficacia, de conocimiento del medicamento, preferencia por la vía de administración, gastos indirectos).

Se excluyeron del estudio aquellos pacientes con enfermedades neurológicas, psiquiátricas, neoplasias, trastornos cognitivos, analfabetismo u otra condición que a criterio del investigador pudiere interferir con la compresión y llenado de los cuestionarios.

Dadas las características de los instrumentos nombrados, se utilizó el Cuestionario de adherencia CQR y el de adherencia con DMAR, SMAQ.

\section{Análisis estadístico}

Estimando una proporción de cumplimiento del 60\% para aquellos sin cobertura (casos), similar a lo publicado, y del $75 \%$ para pacientes con cobertura social (controles), similar a países desarrollados, se realizó un cálculo de tamaño de la muestra para obtener un nivel de significación o p <0,05 y una potencia del $80 \%$. Se estimó un total de 335 pacientes encuestados, teniendo en cuenta que podrían perderse un $10 \%$ de las encuestas por respuestas inadecuadas o cuestionarios incompletos.

Para los resultados de la estadística descriptiva, las variables cuantitativas fueron expresadas como media, IC 95\% y desvíos estándar o intervalos intercuartilos, según correspondiera a datos paramétricos o no, respectivamente. Las variables cualitativas se expresaron como distribuciones de frecuencia y porcentajes. Para el análisis de cumplimiento entre los grupos según cobertura social se aplicó la prueba de Chi cuadrado. Para la evaluación de factores asociados a falta de adherencia al medicamento biológico se realizó un análisis de regresión logística. Se consideró significancia $p<0,05$.

\section{Aspectos éticos}

Los pacientes fueron invitados a participar por personal no médico de cada centro participante. Según pautas éticas internacionales ${ }^{22}$. Las normas de la ley de Protección de datos personales (Ley 25.326) no se aplicó ya que el estudio se basa en una encuesta de opinión y los datos recogidos no pueden atribuirse a una persona determinada ${ }^{23}$.

\section{Resultados}

Se encuestaron 345 pacientes, de los cuales 29 fueron excluidos $(8,4 \%)$ por no reunir los criterios de selección. El $86 \%$ (271) fueron mujeres, la edad promedio fue de 55 años (43-67) y el tiempo de evolución de la AR de 15 años (6-24). Nivel de educación: 144 (46\%) estudios primarios, 104 (33\%) secundarios, 68 (21\%) universitarios. La procedencia de los pacientes se puede observar en la Figura 1 y la cobertura de salud en la Figura 2.

Los pacientes se encontraban tratados con una mediana de 3 (RIQ 3-4) medicamentos para la AR y además recibían una mediana de 2 (RIQ 0-3) drogas por otras patologías, con un número mediano de medicamentos por cualquier causa de 5 (RIQ 4-7).

La frecuencia y porcentaje de los biológicos empleados se muestran en la Figura 3. La mediana de tiempo de tratamiento con DMAR biológica fue 24 meses (RIC 12-48). 


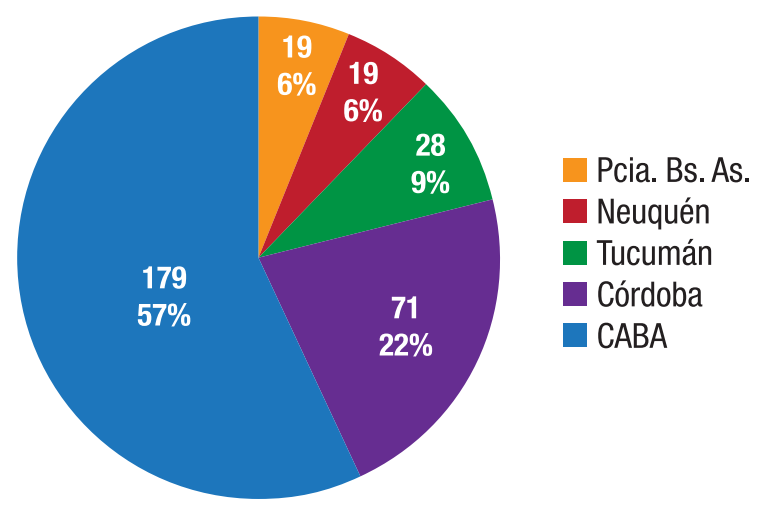

Figura 1. Procedencia de los pacientes con AR.

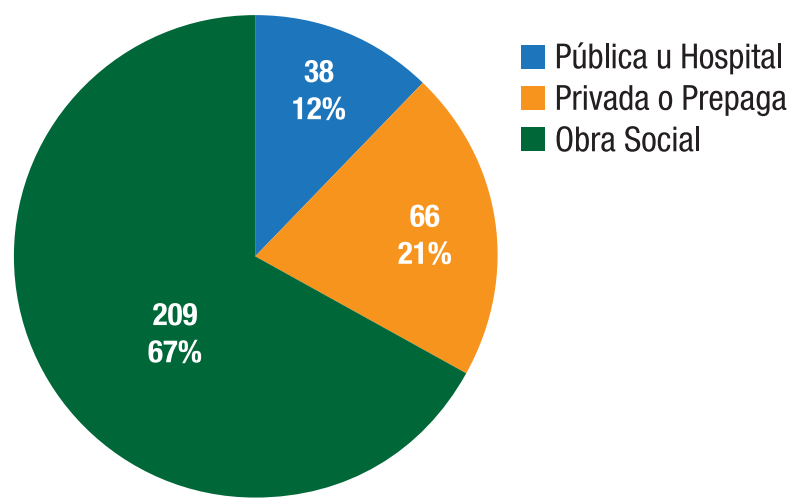

Figura 2. Cobertura de salud de los pacientes con AR.

Forma de aplicación del medicamento biológico El 38\% (120) de los pacientes recibió la medicación en un centro bajo supervisión médica o se lo administró un médico; el 38\% (118) se autoadministró y en un 24\% (76) se lo aplicó una enfermera o lo recibió en una farmacia.

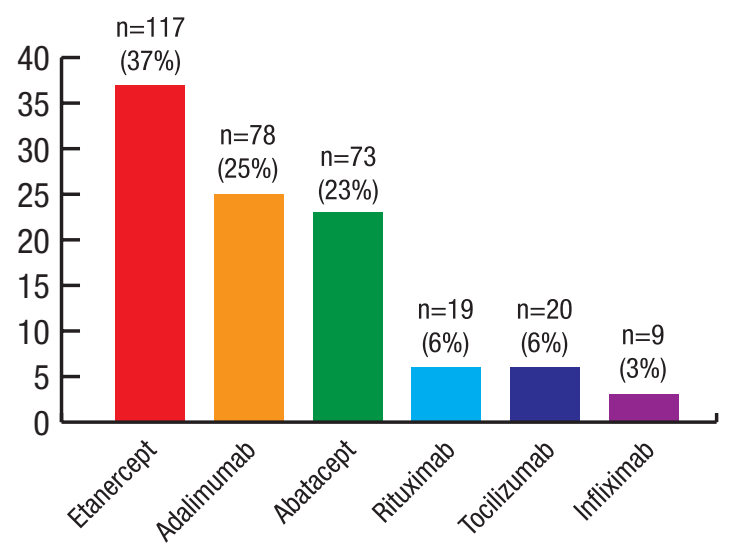

Figura 3. Terapia biológica de los pacientes con AR.
Preocupaciones del paciente relacionadas al tratamiento biológico

La vía de administración no le ocasiona molestias de importancia al 63\% (200) de los pacientes, mientras que el 29\% (91) manifestó molestias leves. La frecuencia de administración no le representa inconvenientes al 74\% (235) mientras el 19\% (61) desearía que sea menos frecuente. El 82\% (274) de los pacientes piensan que la medicación biológica es segura (seguridad). El 30\% (88) de los pacientes esperan una rápida respuesta al tratamiento biológico mientras que el 70\% restante (203) no le preocupa la rapidez de respuesta en tanto sea eficaz (eficacia). Un 9\% (29) no notó mejoría suficiente pero piensa que va a mejorar y un 4\% (14) no notó mejoría y piensa dejar el tratamiento. Las preferencias sobre la forma de aplicación del biológico se observan en la Figura 4.

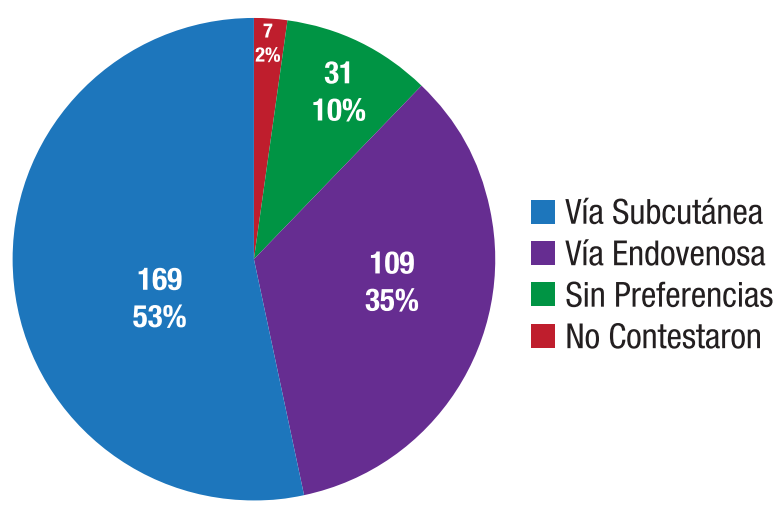

Figura 4. Preferencia de los pacientes en relación a la vía de administración del biológico.

Adherencia al tratamiento farmacológico con DMAR no biológicas

Mediante el cuestionario SMAQ se observó una adherencia del 50\% (159 pacientes), siendo los motivos de falta de cumplimiento los siguientes: el 25\% dejó de tomar alguna vez la medicación para su AR, un $12 \%$ olvidó tomar la medicación los fines de semana, la mediana de dosis perdidas en la última semana fue de 1 (RIC 1-4) y el 34\% refirió haber estado un día completo sin medicación en los últimos 90 días.

El Cuestionario CQR tuvo un puntaje mediano de 78 puntos (RIC 67-86). El 47\% (147 pacientes) fueron adherentes $(\mathrm{CQR}>80)$.

Adherencia al tratamiento farmacológico con DMAR biológicas

Sobre los 316 pacientes incluidos, 151 (48\%) refirieron no haber tenido ningún retraso, pérdida o adelanto de la dosis del biológico en los últimos 6 meses de tratamiento. 
Considerando la falta de cumplimiento (no adherencia) al retraso, pérdida o adelanto en la dosis del biológico en los últimos 6 meses de tratamiento: 165 pacientes (52\%) fueron NO adherentes. Las causas se dividen en: 146 (46\%) pérdida de al menos una dosis del biológico con una mediana de dosis perdidas de 2 (RIQ: 1-3); 117 (37\%) tuvo al menos un retraso en las dosis del biológico y 8 (2\%) adelantó la dosis.

\section{Motivos de pérdida de dosis del biológico}

El 78\% (115/146) atribuyó las pérdidas de dosis a causas administrativas. El 49\% (72/146) a causas no administrativas, dentro de las cuales: 24 (9,6\%) pacientes refirió "por olvido"; 4 (1,4\%) "porque me sentía bien"; 15 (5,4\%) por "efectos adversos" (sin incluir infecciones); 6 (2,4\%) "por infecciones"; 2 (0,7\%) "por cirugias"; 21 (8,2\%) por "otras causas" sin especificar.

\begin{tabular}{|c|c|c|c|}
\hline & ADHERENTE & NO ADHERENTE & $p$ \\
\hline & Media (DE) & Media (DE) & \\
\hline Edad & $54 \pm 11$ & $56 \pm 13$ & 0,99 \\
\hline Tiempo de evolución de la AR & $16 \pm 10$ & $14 \pm 9$ & 0,2 \\
\hline № de medicamentos & $5,4 \pm 2$ & $5,9 \pm 2$ & 0,1 \\
\hline Tiempo de tratamiento & $31 \pm 25$ & $35 \pm 30$ & 0,17 \\
\hline Cuestionario CQR & $74 \pm 15$ & $73 \pm 17$ & 0,5 \\
\hline $\begin{array}{l}\text { Gastos asociados a la administración } \\
\text { del biológico }\end{array}$ & $32 \pm 8,7$ & $30 \pm 8,6$ & 0,9 \\
\hline & $n(\%)$ & $\mathrm{n}(\%)$ & \\
\hline Medicina prepaga & $43(65 \%)$ & $23(34 \%)$ & \multirow{3}{*}{0,002} \\
\hline Obra social & $94(45 \%)$ & $115(55 \%)$ & \\
\hline Medicina pública & $12(32 \%)$ & $26(68 \%)$ & \\
\hline Bajo nivel de educación & $71(46 \%)$ & $73(44 \%)$ & 0,7 \\
\hline Molestias por vía de administración & $4(2,7 \%)$ & $6(3,9 \%)$ & 0,3 \\
\hline Algún olvido en la toma de DMAR & $26(33 \%)$ & $52(67 \%)$ & 0,002 \\
\hline Esperar un efecto rápido del biológico & $31(34 \%)$ & $58(66 \%)$ & 0,003 \\
\hline No notar mejoría con el biológico & $8(18 \%)$ & $35(82 \%)$ & 0,005 \\
\hline Autoadministración & $55(47 \%)$ & $63(53 \%)$ & 0,7 \\
\hline No adherencia a DMAR (SMAQ) & $63(40 \%)$ & $94(60 \%)$ & 0,005 \\
\hline
\end{tabular}

Tabla 1. Comparación entre grupo de pacientes con y sin adherencia a la terapia biológica, definida como si el paciente no cumplió con la prescripción por cualquier motivo y debió retrasar, adelantar o perder una dosis del biológico.

En la Tabla 1 se muestra la comparación entre grupo de pacientes adherentes y no adherentes al tratamiento con DMAR biológicas. Los factores asociados a falta de cumplimiento de la terapia biológica fueron: la cobertu- ra médica (Pública vs. Prepaga OR: 4,6; Obra Social vs. Prepaga OR: 2,3), que el paciente no haya notado mejoría con la terapia biológica (OR: 4), que el paciente refiere algún olvido en la toma de medicamentos DMAR (OR: 2,8) y esperar una rápida respuesta al tratamiento biológico (OR: 2).

\begin{tabular}{|l|c|c|c|c|}
\hline & B & S.E. & $\boldsymbol{p}$ & OR \\
\hline No notar mejoría con el biológico & $\mathbf{1 , 3 9 6}$ & $\mathbf{0 , 5 2 5}$ & $\mathbf{0 , 0 0 8}$ & $\mathbf{4 , 0 4 0}$ \\
\hline Algún olvido en la toma de DMAR & $\mathbf{1 , 0 3 1}$ & $\mathbf{0 , 3 0 8}$ & $\mathbf{0 , 0 0 1}$ & $\mathbf{2 , 8 0 4}$ \\
\hline Esperar un efecto rápido del biológico & $\mathbf{0 , 7 1 2}$ & $\mathbf{0 , 2 8 6}$ & $\mathbf{0 , 0 1 3}$ & $\mathbf{2 , 0 3 8}$ \\
\hline Medicina Pública vs. Prepaga & $\mathbf{1 , 5 4 7}$ & $\mathbf{0 , 4 7 3}$ & $\mathbf{0 , 0 0 1}$ & $\mathbf{4 , 6 9 6}$ \\
\hline Obra Social vs. Prepaga & $\mathbf{0 , 8 5 2}$ & $\mathbf{0 , 3 4 1}$ & $\mathbf{0 , 0 1 2}$ & $\mathbf{2 , 3 4 5}$ \\
\hline
\end{tabular}

Tabla 2. Factores asociados a la no adherencia a la terapia biológica, en análisis multivariado.

\section{Discusión}

La falta de adherencia es un fenómeno conocido en todas las áreas terapéuticas, que contribuye a una respuesta inadecuada o al fracaso del tratamiento, al empeoramiento y la progresión de la enfermedad y a las recaídas o reaparición de los síntomas. Estos resultados clínicos subóptimos por falta de adherencia conducen a cambios innecesarios de regímenes de tratamiento, a procesos más complejos de diagnóstico y pérdidas económicas ${ }^{24,25,26}$.

En un estudio reciente que empleó el cuestionario CQR para el cumplimiento al tratamiento con DMAR de 228 pacientes con Artritis Reumatoidea, un 68\% fueron adherentes y la falta de adherencia se asoció a la duración de enfermedad, el número de efectos adversos percibidos y creencia en la necesidad del tratamiento ${ }^{16}$.

En nuestro estudio observamos una falta de cumplimiento a la terapia con DMAR del 47-50\% empleando dos cuestionarios validados y del $52 \%$ a la terapia biológica (definido como retraso, pérdida o adelanto en la dosis del biológico).

Una revisión de la literatura conducida por Harrold y Andrade encontró 11 estudios que evalúan la adherencia a tratamientos farmacológicos para condiciones crónicas reumáticas incluyendo AR, artritis inflamatoria, polimialgia reumática, artritis idiopática juvenil y lupus sistémico eritematoso ${ }^{27}$. Sin embargo, la revisión no se enfocó en la adhesión específica a DMAR biológicas. La mayor parte de los estudios que evaluaron esta problemática lo hicieron en relación a AINE y DMAR tradicionales, por lo general con un bajo tamaño de la muestra. Similar a otros 
campos de la medicina, la revisión concluyó que había inconsistencia en definiciones y medida de adherencia usada. Por lo tanto, poblaciones diferentes de pacientes fueron evaluadas con los diferentes métodos, lo que hace difícil obtener una conclusión de estos estudios ${ }^{9,18}$.

La introducción de bases de datos electrónicas (por ejemplo, bases de datos del seguro médico en Norteamérica, registros de biológicos en Europa y Norteamérica) hizo posible un mejor análisis, tanto del cumplimiento como de la persistencia con DMAR biológicas ${ }^{8}$. Sin embargo, los estudios realizados sobre adherencia a biofármacos para AR siguen siendo escasos.

Muchos estudios evalúan la persistencia como sinónimo de sobrevida del tratamiento y la metodología utilizada es el análisis de supervivencia. En ese caso la medida del cumplimiento es la tasa de posesión del medicamento (TPM): número de días que el paciente realmente tiene posesión de la medicación (basada en el rescate de prescripción y el recambio), dividido por los días de tratamiento. Sería de interés incluir ese tipo de análisis en un próximo estudio que haga nuestro grupo. De todas formas, la mayor parte de los estudios nombrados fueron retrospectivos, basados en bases de datos europeas y de Estados Unidos con tamaños muestrales entre 304 y 14.932 pacientes $^{28,29}$.

Grijalva y cols. investigaron tanto el cumplimiento (definido como adherencia y medido como TPM) como la persistencia en un análisis retrospectivo de 12 diferentes regímenes de tratamiento que incluía tanto DMAR tradicionales como biológicas en monoterapia y terapia combinada, en una base de datos retrospectiva de aproximadamente 15.000 pacientes con AR (período 1995-2004). Considerando al metotrexato como droga de referencia, se encontró que el cumplimiento fue más alto para Infliximab, Etanercept y Adalimumab como monoterapia y más bajo para terapia combinada de metotrexato con cualquiera de los medicamentos biológicos mencionados. Con respecto a las DMAR tradicionales, leflunomida tuvo la tasa más alta de cumplimiento, mientras que las más bajas fueron las de sulfasalazina e hidroxicloroquina en comparación con metotrexato ${ }^{8}$.

Harley y cols., mediante un análisis retrospectivo de una base de datos de pacientes con AR (período 19982000), informaron el cumplimiento para etanercept, infliximab y metotrexato como el número de administraciones o prescripciones dividido por el número esperado. El cumplimiento fue definido como al menos el $80 \%$ de las administraciones o prescripciones esperadas y se encontró que éstas fueron significativamente más bajas para etanercept y metotrexato que para infliximab ${ }^{30}$.
La persistencia es comunicada como el promedio de tiempo o el porcentaje de pacientes que aún reciben la droga en un período de tiempo determinado. Al diferenciar la persistencia entre los tres inhibidores del TNF alfa, los resultados no fueron concluyentes. Tres artículos concluyen que la persistencia con Infliximab fue mayor que con Adalimumab o Etanercept ${ }^{31,32,33}$, mientras cinco artículos encontraron lo opuesto ${ }^{34,35,36,37}$ y cuatro estudios no encontraron diferencias ${ }^{38,39,40,41}$.

Hubo un solo estudio que informó persistencia con DMAR tradicionales, metotrexato y uno en comparación con leflunomida. Mientras que la tasa de persistencia absoluta con metotrexato no fue comunicada, sólo el $22 \%$ de los pacientes permanecieron con leflunomida luego de 20 meses de iniciado el tratamiento ${ }^{8,32}$. El uso concomitante de metotrexato proporcionó una mayor sobrevida que el empleo de otras DMAR asociadas ${ }^{29}$.

\section{Conclusiones}

En pacientes con AR que se encuentran recibiendo terapias biológicas, el cumplimiento al régimen terapéutico es bajo, tanto para DMAR tradicionales como DMAR biológicas. Los factores asociados al no cumplimiento de la terapia biológica son el tipo de cobertura médica, que el paciente no haya notado mejoría, la esperanza de una rápida respuesta al tratamiento y la falta de adherencia a DMAR. Serán necesarios más estudios nacionales para seguir investigando la adherencia a estos tratamientos.

\section{Bibliografía}

1. Gerth WC. Compliance and persistence with newer antihypertensive agents. Curr Hypertens Rep 2002;4(6):424-33.

2. Knobel H, Alonso J, Casado JL, Collazos J, González J, Ruiz I, et al. Validation of a simplified medication adherence questionnaire in a large cohort of HIV-infected patients: the GEEMA Study. AIDS. 2002; 16 (4): 605-13.

3. Rogers PGBW. Prescription medication compliance: a review of the baseline of knowledge. A report of the national council on patient information and patient education. Journal of Pharmacoepidemiology and Drug Safety 1995;2:3-36.

4. Sackett, DLHR. Compliance with therapeutic regimens. Baltimore: Johns Hopkins University Press; 1976.

5. Buchbinder R, Hall S, Sambrook PN, et al. Metho- 
trexate therapy in rheumatoid arthritis: a life table review of 587 patients treated in community practice. J Rheumatol 1993;20(4):639-44.

6. World Health Organization: World Health Organization: Noncommunicable Diseases and Mental Health - Adherence to Longterm Therapies: Policy for Action. Meeting Report 4-5 June, 2001. Geneva: WHO/MNC/CCH/01.02; 2001.

7. Osterberg L, Blaschke T. Adherence to medication. N Engl J Med 2005;353:487-97. Review on patient adherence in general.

8. Grijalva CG, Chung CP, Arbogast PG, et al. Assessment of adherence to and persistence on disease-modifying antirheumatic drugs (DMARDs) in patients with rheumatoid arthritis. Med Care 2007;45(10 Suppl 2):S66-76.

9. Hughes D, Cowell W, Koncz T, Cramer J. International Society for Pharmacoeconomics \& Outcomes Research Economics of Medication Compliance Working Group. Methods for integrating medication compliance and persistence in pharmacoeconomic evaluations. Value Health 2007;10:498-509.

10. Smolen JS, Aletaha D, Koeller M, et al. New therapies for treatment of rheumatoid arthritis. Lancet 2007;370:1861-74.

11. Bingham CO III. Emerging therapeutics for rheumatoid arthritis. Bull NYU Hosp J Dis 2008;66:210-15.

12. Furst DE, Keystone EC, Kirkham B, et al. Updated consensus statement on biological agents for the treatment of rheumatic diseases, 2008. Ann Rheum Dis 2008;67(Suppl 3):iii2-25.

13. Pincus T, Stein CM. Why randomized controlled clinical trials do not depict accurately long-term outcomes in rheumatoid arthritis: some explanations and suggestions for future studies. Clin Exp Rheumatol 1997;15(17):S27-38.

14. Zink A, Strangfeld A, Schneider M, et al. Effectiveness of tumor necrosis factor inhibitors in rheumatoid arthritis in an observational cohort study: comparison of patients according to their eligibility for major randomized clinical trials. Arthritis Rheum 2006;54(11):3399-407.

15. Revicki DA, Frank L. Pharmacoeconomic evaluation in the real world. Effectiveness versus efficacy studies. Pharmacoeconomics 1999;15:423-34.

16. van den Bemt BJF, van Lankveld GJMW. How can we improve adherence to therapy by patients with rheumatoid arthritis? Nat Clin Pract Rheumatol 2007;3:681.

17. BART J.F. van den BEMT, FRANK H.J. van den HOOGEN, BART BENRAAD, YECHIEL A. HEKSTER, PIET L.C.M. van RIEL, and WIM van LANKVELD. Adherence Rates and Associations with Nonadherence in Patients with Rheumatoid Arthritis Using Disease Modifying Antirheumatic Drugs. J Rheumatol 2009;36:2164-70.

18. Burrell A, Wong P, Ollendorf D, et al. Defining compliance/adherence and persistence. ISPOR Special Interest Working Group. Value Health 2005;8:A194-195.

19. Koncz T, Pentek M, Brodszky V, Ersek K, Orlewska E, Gulacsi L. Adherence to biologic DMARD therapies in rheumatoid arthritis. Expert Opin Biol Ther. 2010 Sep;10(9):1367-78.

20. de Klerk E, van der Heijde D, Landewé R, van der Tempel H, van der Linden S. The compliance-questionnaire-rheumatology compared with electronic medication event monitoring: a validation study. J Rheumatol. 2003;30(11):2469-75.

21. Arnett FC, Edworthy SM, Bloch DA, McShane DJ, Fries JF, Cooper NS, et al. The American Rheumatism Association 1987 revised criteria for the classification of rheumatoid arthritis. Arthritis Rheum 1988;31(3):315-24.

22. Council for International Organizations of Medical Sciences (CIOMS) in collaboration with the World Health Organization (WHO). International Ethical Guidelines for Epidemiological Studies. Geneva 2008.

23. Ley 25.326 Protección de datos personales. Disposiciones Generales. Principios generales relativos a la protección de datos. Derechos de los titulares de datos. Usuarios y responsables de archivos, registros y bancos de datos. Control. Sanciones. Acción de protección de los datos personales. Sancionada: Octubre 4 de 2000. Promulgada Parcialmente: Octubre 30 de 2000.Senado y Cámara de Diputados de la Nación Argentina reunidos en Congreso.

24. Osterberg L, Blaschke T. Adherence to medication. N Engl J Med 2005;353:487-97. Review on patient adherence in general.

25. Grijalva CG, Chung CP, Arbogast PG, et al. Assessment of adherence to and persistence on disease-modifying antirheumatic drugs (DMARDs) in patients with rheumatoid arthritis. Med Care 
2007;45(10 Suppl 2):S66-76.

26. Hughes D, Cowell W, Koncz T, Cramer J. International Society for Pharmacoeconomics \& Outcomes Research Economics of Medication Compliance Working Group. Methods for integrating medication compliance and persistence in pharmacoeconomic evaluations. Value Health 2007;10:498-509.

27. Harrold LR, Andrade SE. Medication adherence of patients with selected rheumatic conditions: a systematic review of the literature. Semin Arthritis Rheum 2009;38:396-402.

28. Borah BJ, Huang X, Zarotsky V, Globe D. Trends in RA patients' adherence to subcutaneous antiTNF therapies and costs. Curr Med Res Opin 2009;25:1365-77.

29. Curkendall S, Patel V, Gleeson M, et al. Compliance with biologic therapies for rheumatoid arthritis: do patient out-of-pocket payments matter? Arthritis Rheum 2008;59:1519-26.

30. Harley CR, Frytak JR, Tandon N. Treatment compliance and dosage administration among rheumatoid arthritis patients receiving infliximab, etanercept, or methotrexate. Am J Manag Care 2003;9(6 Suppl):S136-43.

31. Tang B, Rahman M, Waters HC, Callegari P. Treatment persistence with adalimumab, etanercept, or Infliximab in combination with methotrexate and the effects on health care costs in patients with rheumatoid arthritis. Clin Ther 2008; 30:1375-84.

32. Yazici Y, Krasnokutsky S, Barnes JP, et al. Changing patterns of tumor necrosis factor inhibitor use in 9074 patients with rheumatoid arthritis. J Rheumatol 2009;36:907-13.

33. Gomez-Reino JJ, Carmona L. BIOBADASER Group. Switching TNF antagonists in patients with chronic arthritis: an observational study of 488 patients over a four-year period. Arthritis Res Ther 2006;8(1):R29.

34. Brocq O, Roux $\mathrm{CH}$, Albert $\mathrm{C}$, et al. TNFalpha antagonist continuation rates in 442 patients with inflammatory joint disease. Joint Bone Spine 2007;74:148-54.

35. Kristensen LE, Saxne T, Nilsson JA, Geborek P. Impact of concomitant DMARD therapy on adherence to treatment with etanercept and Infliximab in rheumatoid arthritis. Results from a six-year observational study in southern Sweden. Arthritis Res Ther 2006;8(6):R174.
36. Hetland ML, Christensen IJ, Tarp U, Dreyer L, Hansen A, Hansen IT, et al. Direct comparison of treatment responses, remission rates, and drug adherence in patients with rheumatoid arthritis treated with adalimumab, etanercept, or infliximab: results from eight years of surveillance of clinical practice in the nationwide Danish DANBIO registry. Arthritis Rheum 2010;62:22-32.

37. Marchesoni A, Zaccara E, Gorla R, et al. TNF-alpha antagonist survival rate in a cohort of rheumatoid arthritis patients observed under conditions of standard clinical practice. Ann NY Acad Sci 2009;1173:837-46.

38. Geborek P, Crnkic M, Petersson IF, et al. Etanercept, infliximab, and leflunomide in established rheumatoid arthritis: clinical experience using a structured follow up programme in southern Sweden. Ann Rheum Dis 2002;61:793-8.

39. Flendrie M, Creemers MC, Welsing PM, et al. Survival during treatment with tumour necrosis factor blocking agents in rheumatoid arthritis. Ann Rheum Dis 2003;62(Suppl 2):ii30-33.

40. Duclos M, Gossec L, Ruyssen-Witrand A, et al. Retention rates of tumor necrosis factor blockers in daily practice in 770 rheumatic patients. Rheumatol 2006;33:2433-8.

41. Zink A, Listing J, Kary S, et al. Treatment continuation in patients receiving biological agents or conventional DMARD therapy. Ann Rheum Dis 2005;64:1274-9. 